\title{
Review on Foreign Object Debris Detection Technologies and Advancement for Airport Safety and Surveillance
}

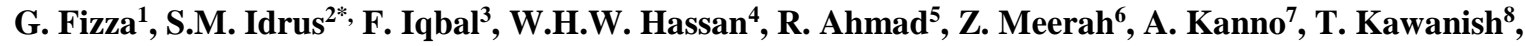 \\ N. Shibagaki', K. Kashima ${ }^{10}$
}

${ }^{1,2,3}$ School of Electrical Engineering, UniversitiTeknologi Malaysia, Malaysia

${ }^{4}$ School of Ocean Engineering, Universiti Malaysia Terengganu, Malaysia

${ }^{5,6}$ Malaysia Airports Holding Berhad, Malaysia

${ }^{7,8}$ National Institute of Information and Communications Technology, Japan

${ }^{9,10}$ Hitachi Kokusai Electric Inc., Japan

Email: ${ }^{2 *}$ sevia@utm.my

Article History: Received: 10 November 2020; Revised: 12 January 2021; Accepted: 27January 2021; Published online: 05April 2021

\begin{abstract}
The rapid growth of air traffic worldwide leads to frequent landing and takeoff of airplanes at busy airports. Therefore, the airport runway must be safe and clean from any tiny size and shape of foreign object debris that is very hard to detect by visual inspection. For successful and fast removal of foreign object debris, an efficient system capable of precisely detecting foreign object debris is required. This paper presents a review of the available technologies to detect foreign object debris and compares commercially available foreign object debris detection technologies. The review has revealed that millimeter-wave radar technology is one of the most efficient foreign object debris detection techniques as it is weather resilient. Furthermore, millimeter-wave radar integrated with radio over fiber technology provides a cost-effective solution to detect foreign object debris within 10 seconds at $96 \mathrm{GHz}$ frequency. A pilot test has been carried out at Narita International Airport, Japan and Kuala Lumpur International Airport, Malaysia, to detect foreign object debris either in a mobile or immobile position.
\end{abstract}

\section{Keywords:Foreign object debris, radar over fiber, millimeterwave, optical camera, sensor}

\section{Introduction}

The International Air Transport Association (IATA) has estimated that current developments in air transport could double the number of travellers to 8.2 billion in 2037 [1]. Aircraft departures are anticipated to rise at an average annual rate of 3.6 to 4.1 percent, respectively between 2005 and 2025, from 24.9 million aircraft departures and 30.8 billion aircraft-kilometers flown in 2005 [2]. Hence, air transport accidents are a serious concern for aviation due to the proliferate growth of air traffic. Aircraft accidents have resulted in 514 fatalities in 2018 and 50 fatalities in 2017 [3]. The International Civil Aviation Organization (ICAO) has identified planned commercial air traffic and security events on runways as one of three high-risk accident categories in the ICAO Global Flight Safety Plan. Runway safety is among the top priority in ensuring the safety of air travellers, where one of its big challenges is the detection and classification of runway debris or foreign object debris (FOD).

FOD can be defined as any debris or damage that can lead to aircraft accident [4-5]. The Air France Flight 4590 had run over debris that had fallen from the aircraft that took off about 4 minutes earlier [6]. Another FODrelated accident is reported by the Australian Transport Safety Bureau (ATSB) where the nose wheel tire of a charter flight was punctured by FOD, but the damage was sustained during landing [7]. Recently, the Gates Learjet Corp 35A tires had blown during take off due to FOD on the runway at the Darwin Airport [8]. FOD is not only a direct threat to aircraft operation, but also an indirect hazard due to the delays and disruptions causedby FOD events. FOD costs the aeronautics industry millions of dollars annually, including the cost of engine failures or air frames and indirect revenue cost (e.g., flight delay, cancellations and additional work by employees). The presence of FOD on runways, ramps, aprons or taxiways during the flight operation is dangerous. For safe airport operation, the runway must remain in an uninterrupted operation state. Regular inspection is an essential part of an aerodrome preventive and corrective maintenance program, with the objective of avoiding and eliminating any loose object or debris [9]. Any contaminant (e.g., snow, slush, ice, standing water, mud, dust, sand, oil, rubber deposits) needs to be completely removed from the runway surface as soon as possible, to minimize further accumulation. FOD can damage the aircraft in critical flight phases, which may lead to catastrophic losses as well as increased maintenance and operating costs if not immediately removed. FOD risks can be reduced by prevention, detection, and removal methods. Thus, an efficient system is required to ensure aircraft safety during landing and take off procedure. 
This paper presents a review on FOD detection methods and advancement on FOD technologies for airport safety and surveillance. This paper further divided into four sections. Section II describes the conventional FOD detection methods and section III covers automated detection methods. In section IV, FOD detection techniques used by airports are discussed. Finally, section V concludes the paper.

\section{Conventional Detection Method}

Runway inspection is usually conducted by visual patrol via vehicles on the runway surface to find and remove any detected FOD, depending on runway availability and operation type. Its may be necessary to hire dedicated personnel to continuously check for FOD, if the presence of any ongoing construction activities. Manual FOD removal is usually sufficient, though special removal equipment is available and suitable for airport operations. Table 1 shows some examples of mechanical and non-mechanical FOD removal equipment.

Table 1. FOD Removal Equipment[10]

\begin{tabular}{ll}
\hline Mechanical & Non Mechanical \\
\hline PowerSweepers & Friction MatSweepers \\
VacuumSystems & Magnetic Bars(attached to vehicles) \\
Jet Air Blowers & Rumble strips (also FOD Shakers) \\
\hline
\end{tabular}

Mechanical equipment use powered equipment to remove, retrieve or contain FOD for proper disposal, where the equipment size can vary from small thrust units to large scale truck-mounted systems. Non-mechanical equipment need not be powered, e.g., sweepers, magnetic bars and rumble strips. Although FOD removal equipment has been used in the past, their effectiveness in removing deposits from tires or vehicle undercarriages is often negligible, and the equipment itself can produce FOD if not regularly cleaned. The current best practice for removing tire based FOD is by visually inspecting vehicles at a designated checkpoint and manually remove any detected debris with a hand tool.

\section{Automated Detection Method}

It can be challenging to pinpoint the exact location of any FOD by visual patrol, and manual visual patrol can also be time-consuming. Therefore, more advanced automated methods to detect FOD with higher accuracy is needed [10]. The comparison between manual and automated detection methods is mentioned in Table 2 . Automated FOD detection system can perform 24/7 inspection and detection, avoiding flight delays and runway closures, with clear visibility even at night time and the ability to operate in all weather conditions [11]. Automated detection techniques can be classified into four categories, namely the ultrasonic sensors, camera detection, radar detection and hybrid detection methods, as shown in Figure 1. A general comparison between FOD detection techniques with respect to resolution and coverage area can be illustrated. Higher resolution and wide coverage areas play a vital role in the design of any FOD detection system. A brief overview of the existing system has been described in the following sections.

Table 2. Comparison between Detection Methods

\begin{tabular}{lcc}
\hline Items & ManualDetection & AutomatedDetection \\
\hline Inspection 24/7 & No & Yes \\
FOD detection using visual inspection prone to errors & Yes & No \\
Nighttime inspection & No & Yes \\
Possibility of FODinspectionsundetected & Yes & No \\
Require more persons & Yes & No \\
Detection of tiny objects & No & Yes \\
\hline
\end{tabular}




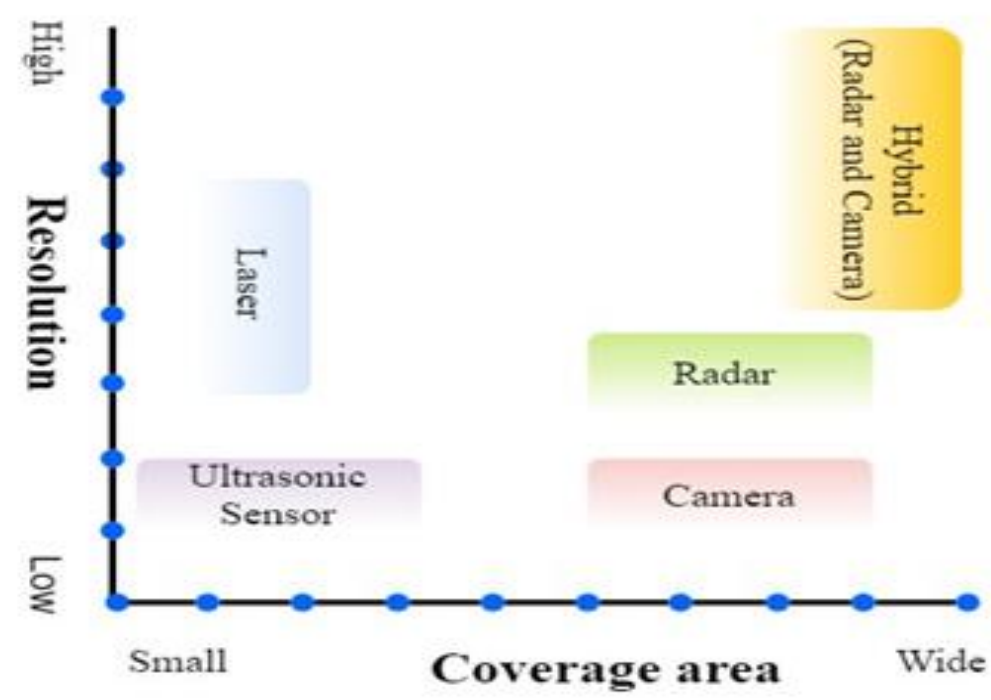

Figure 1. Resolution and Coverage area of Existing FODD system

\subsection{Ultrasonic SensorSystem}

To sense the environment and security systems are the most significant technologies, ultrasonic sensing is one of the ways to sense proximity and detect levels with high reliability and low cost [12-14]. A rover equipped with Light Detection and Ranging (LiDAR) technology and $40 \mathrm{kHz}$ ultrasonic sensors is proposed in [15], where the rover is controlled by Bluetooth android application. LiDAR uses light to scan the path to detect any FOD and calculate the time for the return signal [16]. The proposed system can detect FODs of different sizes with good location accuracy, and the location is shared with the operator, albeit without visual information.

\subsection{Camera DetectionSystem}

Efficient visualization of interference and debris is important to prevent further dangers. For the detection of runway incursion, cracking and obstacles, electro-optical color images and infrared sensor on board the aircraft has been proposed in [17], Image processing techniques have been utilized for detecting FOD andcharacterizing the pavement distress,e.g., [18]proposed a video-based FOD detection setup via a single camera sensor installed on the runway at Nanyang International Airport, where image change algorithm is used to successfully detect FOD of $4 \mathrm{~cm}$ in size [19]. Machine vision is another approach used for runway identification. However, camera detection systems do face visibility difficulties due to weather conditions, fog and darkness.

\subsection{Radar DetectionSystem}

Radar is widely used in public safety and defense as the primary means of detection and surveillance. A miniature radar system with very high resolution is desirable to locate FOD at high precision under all weather conditions.Radar can cover a wide area compared to ultrasonic sensors and lasers. The millimeter-wave technology is a suitable candidate for high-resolution FOD detection [20] since the millimeter-wave radar can operate under both day and night conditions and is very adaptable to bad weather conditions. Millimeter-wave radars have high positioning, sensitivity to small FODs and accuracy in terms of distance and angle,e.g., using Frequency Modulated Continuous Wave (FMCW) radar at millimeter-wave frequencies.FMCW radar has been used to achieve high resolution at $73-80 \mathrm{GHz}$ and $94-95 \mathrm{GHz}[18]$.

\subsection{Hybrid Detection Systems}

The advantages of the radar in shape detection and image in appearance detection have to lead to a hybrid detection system, e.g., using millimeter-wave radar with infrared image sensors [21].Another technique for detecting runway incursion and obstacles, electro-optical color images and an infrared sensor onboard the aircraft is proposed with significant results in day/night and low visibility condition.FOD's visibility and detection have been improved using Radio over Fiber (RoF) technology [22-23].

4. Commercially Available Latest Fod Detection System 
Examples of existing FOD detection systems are Tarsier millimeter-wave radar, hybrid electro-optical millimeter-wave radar with a video camera,intelligent high-resolution cameraand mobile vehicle-mounted millimeter-wave radar. These four FOD detection systems have been tested and approved by the Federal Aviation Administration (FAA). These systems reported are capable of improved runway safety; however,the relatively require high investments [24].General comparison of the system capability is shown in Table 3, including millimeter-wave radar over fiber FOD detection system manufactured by Hitachi Kokusai Electric from Japan[25].Operating millimeter-wave radar for FOD detection is favorable due to its weather-resistant properties. While installing and maintaining the linear cell FOD detection system utilizing RoF integrated with FMCW radar does not require runway closure, it can be considered the most practical and cost-effective solution for the busy airport.The Hitachi's FOD detection system has been installed and currently under field trial at Narita International Airport (NIA) Japan and Kuala Lumpur International Airport (KLIA) Malaysia.

The high precision FODDS consists of $96 \mathrm{GHz}$ frequency radars supported with high-resolution optical cameras to ensure high-quality images, real-time data collection, expediting the investigation process in identifying FOD sources and facilitating fast runway clearing. The specification of the FOD detection system is listed in Table 4 [25].

Figure 2 illustrates the concept of radar over the fiber FOD detection system. The radars along the runway are connected to the airport operation center through the optical fiber cables which are installed in the runway area. The optical frequency modulated continuous wave signals are generated by the optical source and electrical signals at the control center and are optically amplified and distributed via the optical divider to radars. The control center receives the signals from each radar, and the signals are processed, displayed and stored in the server at the monitoring center.

Table 3. Comparison between implemented FOD detection Systems. [24]

\begin{tabular}{|c|c|c|c|c|c|}
\hline $\begin{array}{l}\text { Product } \\
\text { (Manufacturer) }\end{array}$ & Technology & Frequency & Max Range & $\begin{array}{l}\text { Range } \\
\text { Resolution }\end{array}$ & $\begin{array}{l}\text { System } \\
\text { Throughput }\end{array}$ \\
\hline $\begin{array}{l}\text { FODetect } \\
\text { (Xsight System) }\end{array}$ & $\begin{array}{l}\text { mmWave radar with } \\
\text { optical camera }\end{array}$ & $77 \mathrm{GHz}$ & $50 \mathrm{~m}$ & - & $4 \min$ \\
\hline $\begin{array}{l}\text { Moog Tarsier } \\
\text { (Qinetiq) }\end{array}$ & mmWave radar & $94 \mathrm{GHz}$ & $1500 \mathrm{~m}$ & $30 \mathrm{~cm}$ & $72 \mathrm{sec}$ \\
\hline $\begin{array}{l}\text { iFerret } \\
\text { (Stratech) }\end{array}$ & optical camera & - & & - & $70 \mathrm{sec}$ \\
\hline $\begin{array}{l}\text { FOD finder } \\
\text { (Trex Enterprises) }\end{array}$ & $\begin{array}{l}\text { Mobile mmWave } \\
\text { radar }\end{array}$ & $78-81 \mathrm{GHz}$ & $140 \mathrm{~m}$ & - & $45 \mathrm{sec}$ \\
\hline $\begin{array}{l}\text { FOD detection } \\
\text { System } \\
\text { (Hitachi } \\
\text { Kokusai Electric) }\end{array}$ & $\begin{array}{l}\text { mmWave radar over } \\
\text { fiber with HD } \\
\text { Camera }\end{array}$ & $92-100 \mathrm{GHz}$ & $500 \mathrm{~m}$ & $\approx 3 \mathrm{~cm}$ & $10 \mathrm{sec}$ \\
\hline
\end{tabular}

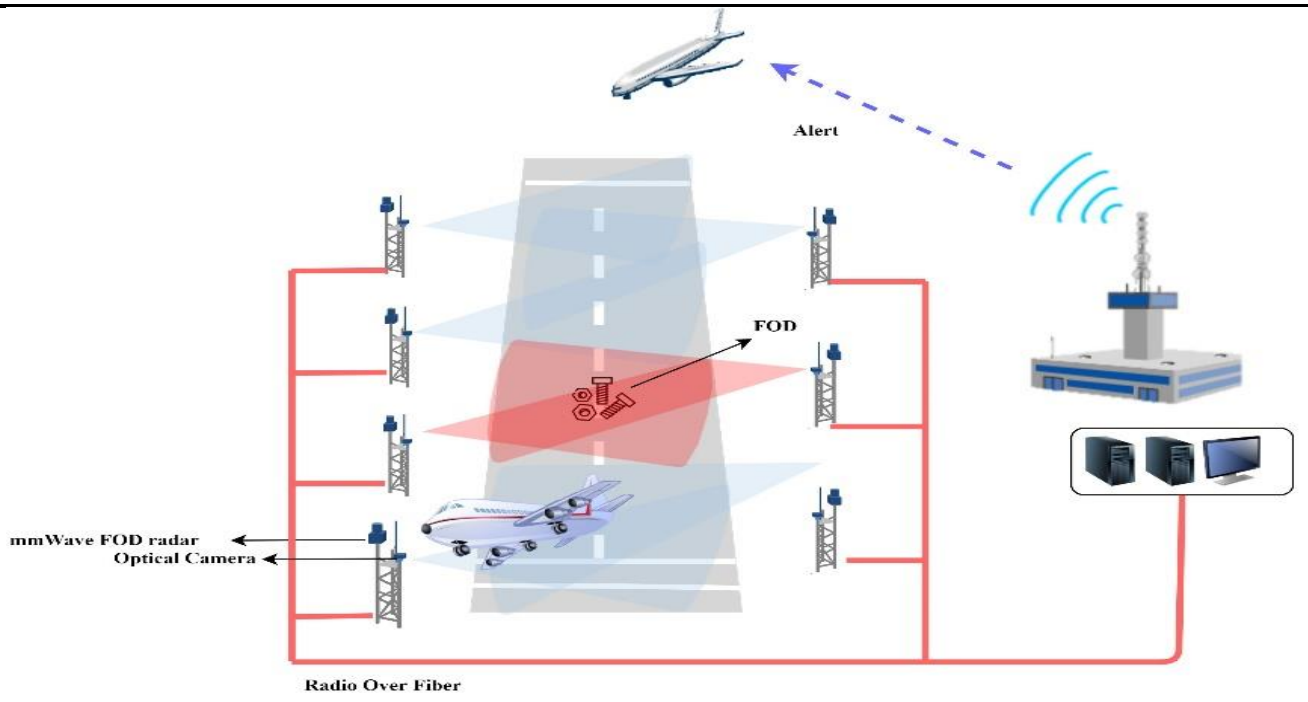

Figure 2.Radar over fiber FOD detection system

Table 4. Specification of FODD system using RoF 
Items

Specifications

mmWaveradar
AntennaGain
Detectionrange
HDcamera

$96 \mathrm{GHz}$

$42 \mathrm{~dB}$

$500 \mathrm{~m}$

Image sensor 1/2.8 3MOS,zoom ratio x25(F.14) OpticalFiber

Single Mode, Wavelength 1310-1500nm

The above FOD detection system is able to detect FOD samples of various sizes, shapes and material at 96 $\mathrm{GHz}$, up to $500 \mathrm{~m}$ range from radar head during the clear sky and $200 \mathrm{~m}$ under $20 \mathrm{~mm} / \mathrm{h}$ rainfall. Under $20 \mathrm{~mm} / \mathrm{h}$ rainfall, it was observed that some scattering appears in the reading due to rain puddles around the FOD. The smallest FOD detected so far during the field trial at KLIA runway is a $1 \mathrm{~cm}$ asphalt. The FOD detection system can detect very small FOD with high accuracy within 10 seconds, whereas other detection systems generally have a response time of more than 45 seconds. The linear cell FOD detection system installed at KLIA Malaysia and NIA Japan is capable of rapid alert by radar sensing with high accuracy while aircrafts takeoff or landing.

\section{Conclusion}

This paper reviewed FOD detection techniques for airport runways. An automated system is expected to replace regular manual inspection four times a day (as required by ICAO). In return, the airport operation's efficiency is maximized since there is no interruption from airport closure due to FOD inspection. Among all the reviewed automated FOD systems, it is found that the millimeter-wave radar technique is the most promising solution. The pilot test using this technique at the KLIA airport has shown promising outcomes. The system named high precision FOD detection system is operated with $96 \mathrm{GHz}$ mm-wave radar integrated with a highresolution optical camera, resulting in a high quality of image detected and real-time data collection. The investigation period to identify the FOD source is significantly minimized and consequentially increase the speed's runway clearing process. Thus, a safer airport runway environment is established with the optimized recourses, and the real-time operation of the airport runaways is maximized.

\section{Acknowledgements}

This work is conducted as a part of a collaborative project entitled "FODDS Field Trial at KLIA" funded by the Ministry of Internal Affairs and Communications, Japan, and UniversitiTeknologi Malaysia Internal Grant vote 01M62. The authors would like to thanks UniversitiTeknologi Malaysia (UTM), Malaysia Airport Holding Berhad, Hitachi Kokusai Electric, Waseda University, NICT Japan, ENRI Japan and iSmartUrusSdnBhd for their cooperation in the field experiments.

\section{References}

1. IATA Forecast Predicts 8.2 billion Air Travelers in 2037, International Air Transport Association(IATA),Press Release No. 62,Date: 24 October 2018.

2. Crucial Meausure for Reducing the CFIT Risk,International Civil Aviation Organization (ICAO) Journal,vol. 62, no. 2,March/April 2007.

3. Safety Report, International Civil Aviation Organization (ICAO), Edition 2018.

4. State of Global Aviation Safety,International Civil Aviation Organization (ICAO) safety report,Edition 2019.

5. Foreign Object Debris (FOD) detection research,International Airport Review,Issue 2,2008.

6. Concorde crash kills 113,BBC News,July, 2000.

7. Foreign object debris involving Piper PA-31, Palm Island, Qld,Australian Transport Safety Bureau (ATSB),AB-2018-13,27 November 2018.

8. Foreign object debris involving a Gates Learjet Corp 35A, Darwin, NT,Australian Transport Safety Bureau (ATSB),No.AB-2019-005, 3 February 2019

9. International Standards and Recommended Practices,Annex 14 to the Convention on International Civil Aviation Aerodromes, Volume I,Aerodrome Design and Operations,Eighth Edition, July 2018.

10. Alathari, Bashar, Mohammed Falih Kadhim, Salam Al-Khammasi, and Nabeel Salih Ali. "A framework implementation of surveillance tracking system based on PIR motion sensors." Indonesian Journal of Electrical Engineering and Computer Science 13, no. 1 (2019): 235-242. 
11. Khairi, Mohd Taufiq Mohd, Sallehuddin Ibrahim, Mohd Amri Md Yunus, and Ahmad Ridhwan Wahap. "Detection of foreign objects in milk using an ultrasonic system." Indonesian Journal of Electrical Engineering and Computer Science 15, no. 3 (2019): 1241-1249.

12. Khan, Talha, Muhammad Alam, Kushairy Kadir, Zeeshan Shahid, M. S. Mazliham, Sheroz Khan, and Mohammad Miqdad. "Recognizing Foreign Object Debris (FOD): False Alarm Reduction Implementation.” Indonesian Journal of Electrical Engineering and Computer Science 11, no. 1 (2018): 41-46.

13. Elrayes, Ahmed, Mohamed Hag Ali, Amer Zakaria, and Mahmoud H. Ismail. "Smart airport foreign object debris detection rover using LiDAR technology.” Internet of Things 5 (2019): 1-11

14. Christian, John A., and Scott Cryan. "A survey of LIDAR technology and its use in spacecraft relative navigation." In AIAA Guidance, Navigation, and Control Conference, p. 4641. 2013.

15. National Academies of Sciences, Engineering, and Medicine. 2011. Current Airport Inspection Practices Regarding FOD (Foreign Object Debris/Damage). Washington, DC: The National Academies Press.

16. N. Yamamoto, T. Umezawa, A. Kanno, K. Akahane, and T. Kawanishi, "High-speed photonic device technologies in optical fiber connected millimeter-wave radar system for foreign object debris detection on runways," in 2014 XXXIth URSI General Assembly and Scientific Symposium (URSI GASS), 2014, pp. 1-4: IEEE.

17. Qunyu, Xu, Ning Huansheng, and Chen Weishi. "Video-based foreign object debris detection." In 2009 IEEE International Workshop on Imaging Systems and Techniques, pp. 119-122. IEEE, 2009.

18. Ibrahim, A., M. K. Osman, N. A. M. Yusof, K. A. Ahmad, N. H. Harun, and R. A. A. Raof. "Characterization of cracking in pavement distress using image processing techniques and k-Nearest neighbour." Indonesian Journal of Electrical Engineering and Computer Science 14, no. 2 (2019): 810.

19. Kawanishi, Tetsuya, Atsushi Kanno, Pham Tien Dat, Toshimasa Umezawa, and Naokatsu Yamamoto. "Photonic Systems and Devices for Linear Cell Radar." Applied Sciences 9, no. 3 (2019): 554.

20. Yonemoto, Naruto, Akiko Kohmura, Shunichi Futatsumori, Tetsuji Uebo, and Alexandre Saillard. "Broad band RF module of millimeter wave radar network for airport FOD detection system." In 2009 International Radar Conference" Surveillance for a Safer World"(RADAR 2009), pp. 1-4. IEEE, 2009.

21. Vegula, Satish Kumar, Sudesh K. Kashyap, and N. Shanthakumar." detection of runway and obstacles using electro-optical and infrared sensors before landing." Defence Science Journal 64, no. 1 (2014): 67-76.

22. Futatsumori, Shunichi, Kazuyuki Morioka, Akiko Kohmura, Kunio Okada, and Naruto Yonemoto. "Design and field feasibility evaluation of distributed-type $96 \mathrm{GHz}$ FMCW millimeter- wave radar based on radio-over-fiber and optical frequency multiplier.” Journal of Lightwave Technology 34, no. 20 (2016): 4835-4843.

23. Husaini, M. M. H., C. B. M. Rashidi, S. N. Azemi, S. A. Aljunid, and M. S. Anuar. "Optical millimeter wave generation utilizing stimulated brillouin scattering for radio over fiber system." Indonesian Journal of Electrical Engineering and Computer Science 13, no. 2 (2019): 818-824.

24. Foreign Object Debris,Assembly-37th Session,Agenda Item 30: Runway Safety, Working paper, A37WP/191 TE/106,International Civil Aviation Organization (ICAO), September 2010.

25. Idrus, S. M., F. Iqbal, A. Kanno, K. Akama, T. Kawanishi, N. Zulkifli, A. Ramli et al. "Demonstration of 95 GHz Single RAU Linear Cell Radar Over Fiber and Radar Propagation Study in Malaysia." In 2018 Progress in Electromagnetics Research. 